\title{
A Method to Check whether Human Understands Contents of Learning Using Electroencephalogram
}

\author{
Hisaki Omae*, Shin-ichi Ito*, Momoyo Ito ${ }^{+}$, Minoru Fukumi ${ }^{+}$ \\ Tokushima University \\ 2-1, Minami-Josanjima, Tokushima, 770-8506, Japan \\ *\{c501737050, s.ito\}@tokushima-u.ac.jp \\ $+\{$ momoito, fukumi\}@is.tokushima-u.ac.jp
}

\begin{abstract}
This paper proposes a method to check whether human understands contents of learning using electroencephalogram (EEG). The proposed method consists of three stages; EEG measurement, EEG feature extraction and EEG pattern classification. The EEG feature extraction method (stage) consists of three phases; frequency analysis, band division based on rhythm of brain activities and principal component extraction. The frequency analysis converts an original waveform of EEG into frequency components (bands). In the band division, frequency components were divided into five rhythms ( $\theta$, Low- $\alpha$, High- $\alpha$, Low- $\beta$ and High- $\beta$ waves). In the principal component extraction, we calculate the first and the second principal components using the principal component analysis (PCA). In the EEG pattern classification, support vector machine (SVM) is used to classify EEG patterns based on PCA results. In order to show the effectiveness of the proposed method, we conduct experiments using real EEG data. The experimental results suggest that the most important sensing position to record EEG is F7 when checking whether he/she understands contents of learning using EEG.
\end{abstract}

Keywords: fast Fourier transform, principal component analysis, support vector machine, understanding of learning, electroencephalogram.

\section{Introduction}

Research on Brain Machine Interface (BMI) has been actively conducted in recent years. The BMI was used to control a wheelchair using $\mathrm{EEG}^{(1)}$ and to operate robot arms using $\mathrm{EEG}^{(2)}$. There are lots of EEG analysis techniques to solve any problems for the BMI developments. The EEG feature extraction techniques include frequency analysis ${ }^{(3)}$, band division ${ }^{(4)}$ and $\mathrm{PCA}^{(5)}$. The SVM is used for EEG pattern classification ${ }^{(5)(6)}$. On the one hand, the EEG signals are constructed on the basis of brain activities. Generally, the frontal lobe is associated with "Thinking"(7), The temporal lobe is also associated with "Memory"(8). However, it is not known whether or not human understands the contents of learning. Therefore, in this paper, we check whether human understands contents of learning using EEG and specify the positions in relation to understanding the contents of learning.

\section{Proposed method}

The proposed method is supposed to check whether human understands contents of learning using EEG. It consists of three stages; the EEG measurement, the EEG feature extraction and the EEG pattern classification. The flowchart of the proposed method is shown in Fig. 1.

\subsection{EEG measurement}

In the EEG measurement, an EPOC device developed by EMOTIV SYSTEMS (system development corporation) is used to measure EEG activity. The EPOC uses dry-type sensors and cover 14ch electrodes (AF3, AF4, F7, F8, F3, F4, T7, T8, FC5, FC6, P7, P8, O1 and O2). In addition, the

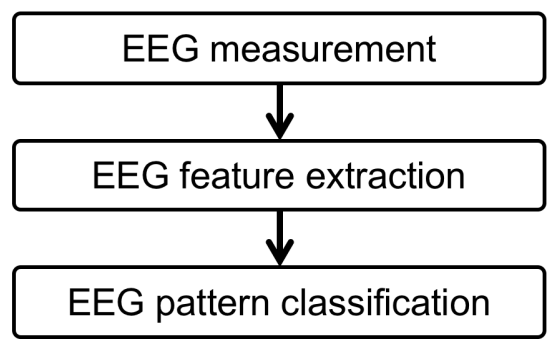

Fig. 1. Flowchart of the proposed method. 


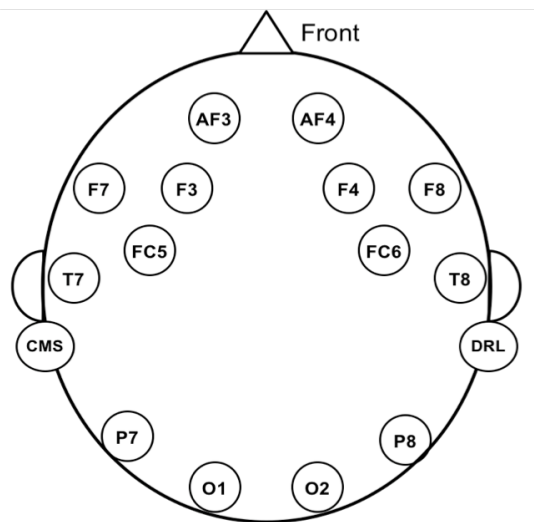

Fig. 2. Extended international 10-20 system.

Table 1. Specification of electroencephalograph (EPOC).

\begin{tabular}{|c|c|}
\hline Number of channels & 14(plus CMS/DRL references) \\
\hline Sampling method & Sequential method, Signal ADC \\
\hline Sampling rate & $128 \mathrm{~Hz}(2048 \mathrm{~Hz}$ Interval) \\
\hline Resolution & $16 \mathrm{bits}$ \\
\hline Bandwidth & $0.2-45 \mathrm{~Hz}$ \\
\hline Filter & Built-in digital fifth order sinc filter \\
\hline Dynamic range & $8,400 \mu \mathrm{V}(\mathrm{pp})$ \\
\hline Coupling Mode & $2.4 \mathrm{GHz}$ band \\
\hline
\end{tabular}

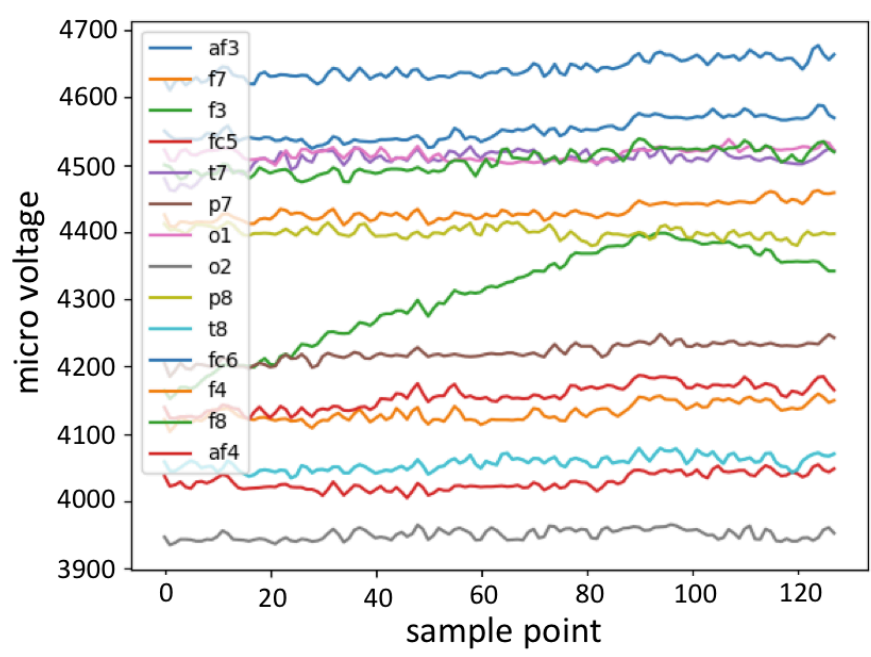

Fig. 3. EEG signals.

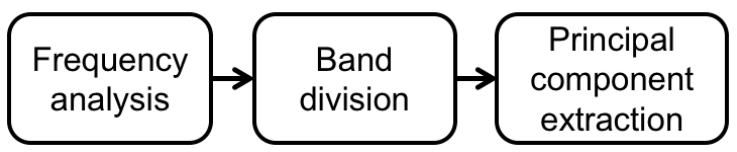

Fig. 4. Flowchart of the EEG feature extraction.

EPOC has a reference electrode on the back of the ear, and the exploration electrode is arranged on the basis of the extended international 10-20 system shown in Fig. 2. The EEG data are sent to a computer through wireless communication. The sampling rate is $128 \mathrm{~Hz}$ as shown in Table 1. We can obtain real EEG signals (shown in Fig. 3).

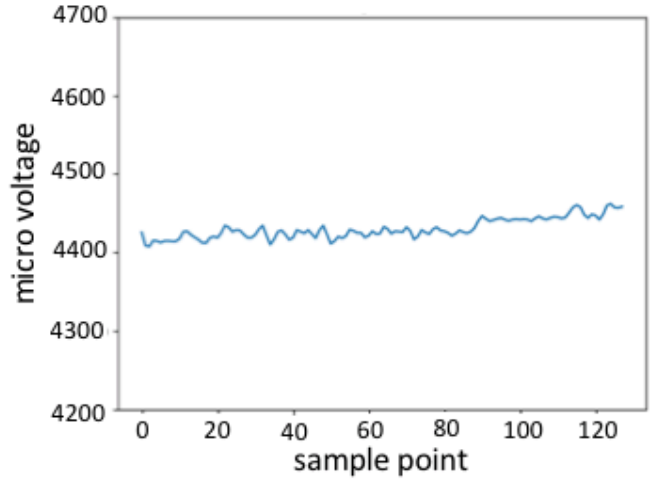

Frequency analysis

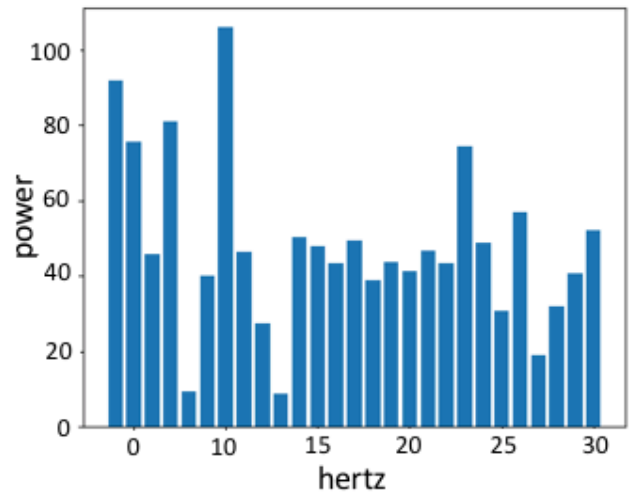

Fig. 5. Sample of frequency analysis.
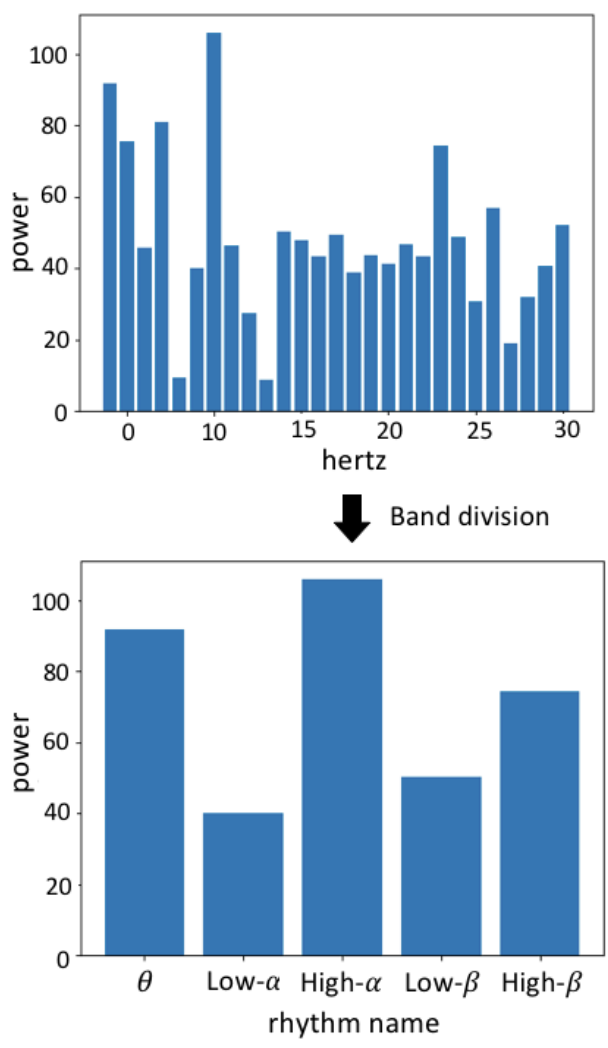

Fig. 6. Sample of band division.

\subsection{EEG feature extraction}

The EEG feature extraction (stage) method consists of 


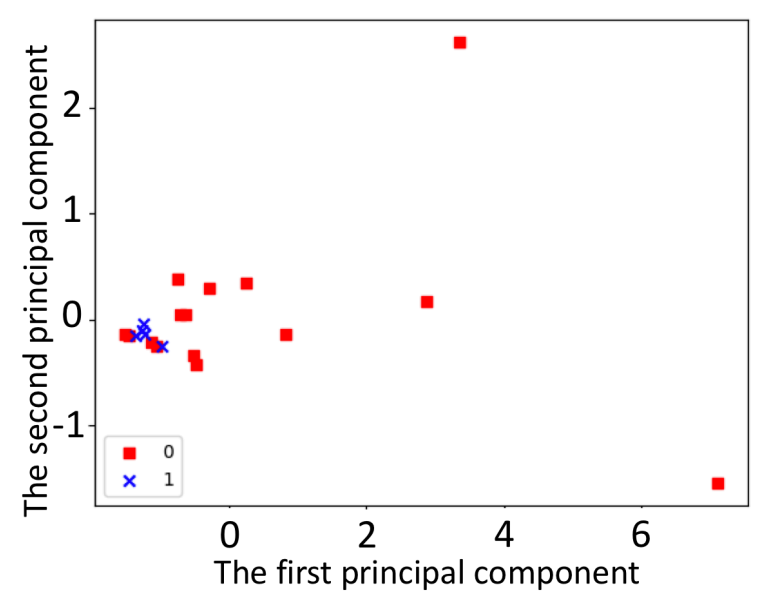

Fig. 7. Sample result of PCA.

three phases; frequency analysis, band division and principal component extraction. The flowchart of the EEG feature extraction is shown in Fig. 4.

In the frequency analysis, the power spectra of EEG data after confirming that a subject understands contents of learning are calculated by fast Fourier transform (FFT). The FFT data cover frequencies from 4 to $30 \mathrm{~Hz}$ at $1 \mathrm{~Hz}$ intervals. The sample of frequency analysis is shown in Fig. 5.

The band division based on rhythm of brain activities divides the power spectra of EEG into $\theta$ wave $(4-7 \mathrm{~Hz}$ ), Low- $\alpha$ wave (8-9 Hz), High- $\alpha$ wave (10-12 Hz), Low- $\beta$ wave (13-17 $\mathrm{Hz})$ and High- $\beta$ wave $(18-30 \mathrm{~Hz})$. The representative value of each band is the maximum value of the power spectra of EEG. The function method is as follows:

$$
P S_{\text {max }}=\max \left(P S_{i}, P S_{i+1}, \cdots, P S_{j}\right)
$$

where $i$ and $j$ are the start and the end of frequency band, respectively. $P S$ s are the power spectra of EEG data. The sample of band division is shown in Fig. 6 .

In the principal component extraction, the first and the second principal components are extracted by PCA. The function of the PCA method is as follow:

$$
Z=w_{1} x^{1}+w_{2} x^{2}+\cdots+w_{m} x^{m}
$$

where $x$ is the corresponding feature vector. In addition, $\sum w_{j}^{2}=1$ and $\mathrm{Z}$ is calculated from the condition of maximum variance. We regard the first and second principal components as the EEG features. A sample result of PCA is shown in Fig. 7.

\subsection{EEG pattern classification}

In the EEG pattern classification, it is checked whether human understands contents of learning by using a classifier. Classes to be classified are two classes (Understanding and Non-Understanding). The SVM is used to classify the EEG features. The SVM calculates the following optimization

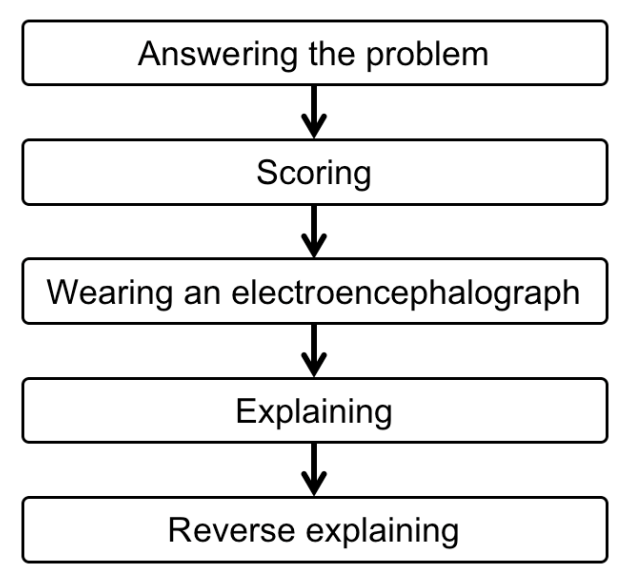

Fig. 8. Time tables of the experiments.

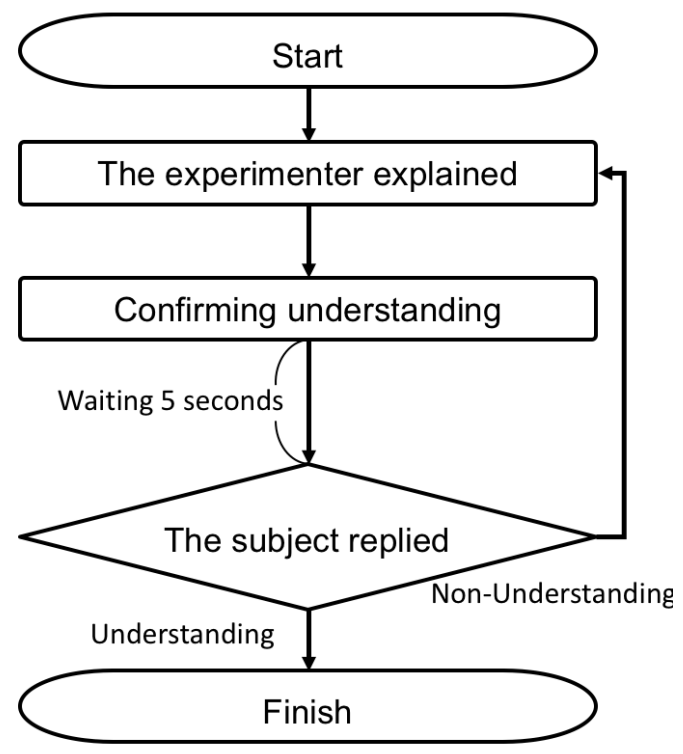

Fig. 9. Flowchart of EEG recording. problem (3)(4).

$$
\begin{gathered}
\arg \min _{w} \frac{1}{2}|w|^{2} \\
t_{n}\left(w^{T} \varphi\left(X_{n}\right)+w_{0}\right)-1 \geq 0
\end{gathered}
$$

where $t$ is a class label. $X$ is a feature vector. $\varphi(\cdot)$ is a kernel function, and this paper adapts the RBF kernel. The function of RBF kernel is as follows:

$$
K\left(x, x^{\prime}\right)=\exp \left(-\frac{1}{2 \sigma^{2}}\left\|x-x^{\prime}\right\|^{2}\right)
$$

where $\sigma$ is standard deviation.

\section{Experiments}

In order to show the effectiveness of the proposed method, this paper conducted the experiments using real EEG data. The subjects in this study comprised two persons: two males (average age 22 years). The subjects conducted the experiment for two days. Figure 8 shows the time table 
of the experiments. In the experiments, he/she answered the test related to the contents of learning. The time limit was set

Table 2. Results of the test related to the contents of learning.

\begin{tabular}{|c|c|c|c|}
\hline & Total & Total U & Total NU \\
\hline Subject A & 20 & 15 & 5 \\
\hline Subject B & 18 & 15 & 3 \\
\hline
\end{tabular}
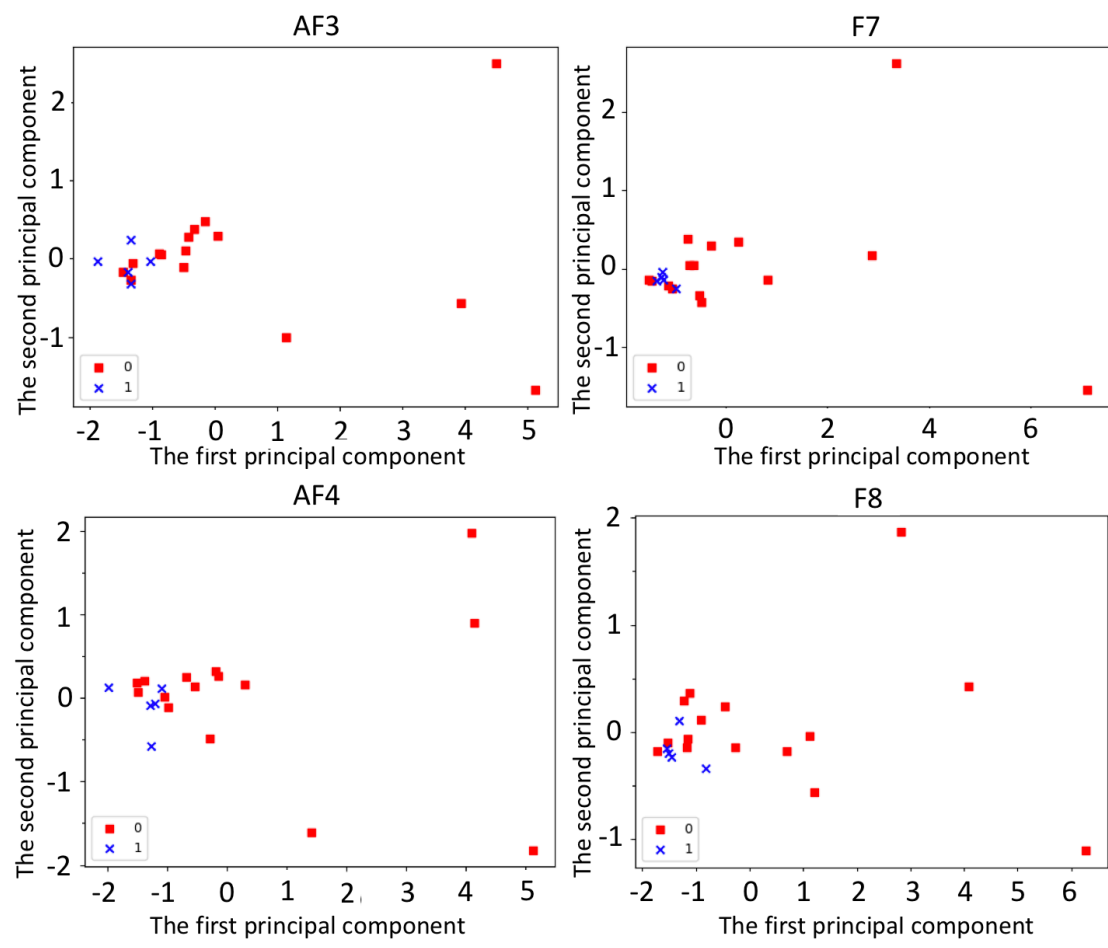

to 20 minutes. The test problem is related to information engineering. Then, the experimenter scored it. Moreover, the subject worn a simple electroencephalograph (EPOC device).

The experimenter expounded at length on the test to him/her. Then the experimenter asked him/her whether understanding the exposition. He/She answered the question after 5 records. If he/she said "No", the experimenter
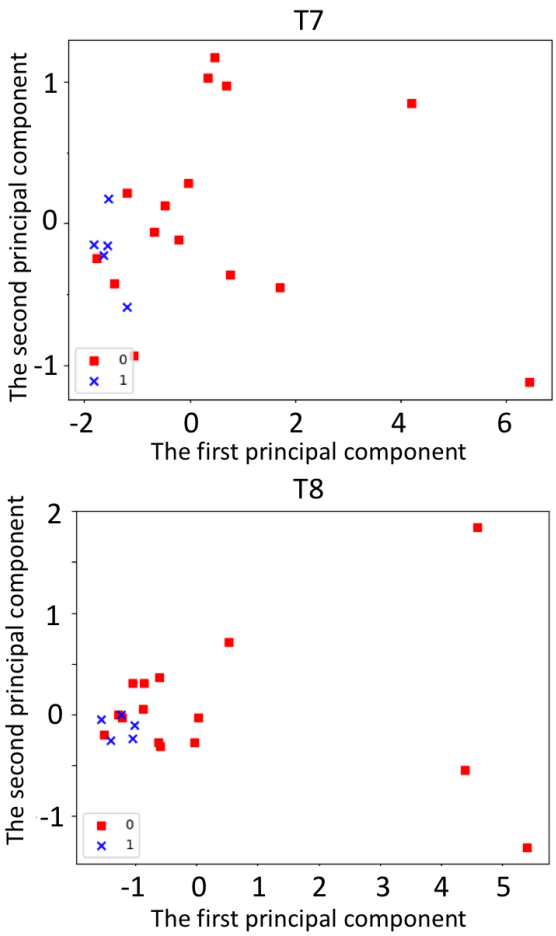

The first principal component .

Fig. 10. Results of subject A obtained using PCA.
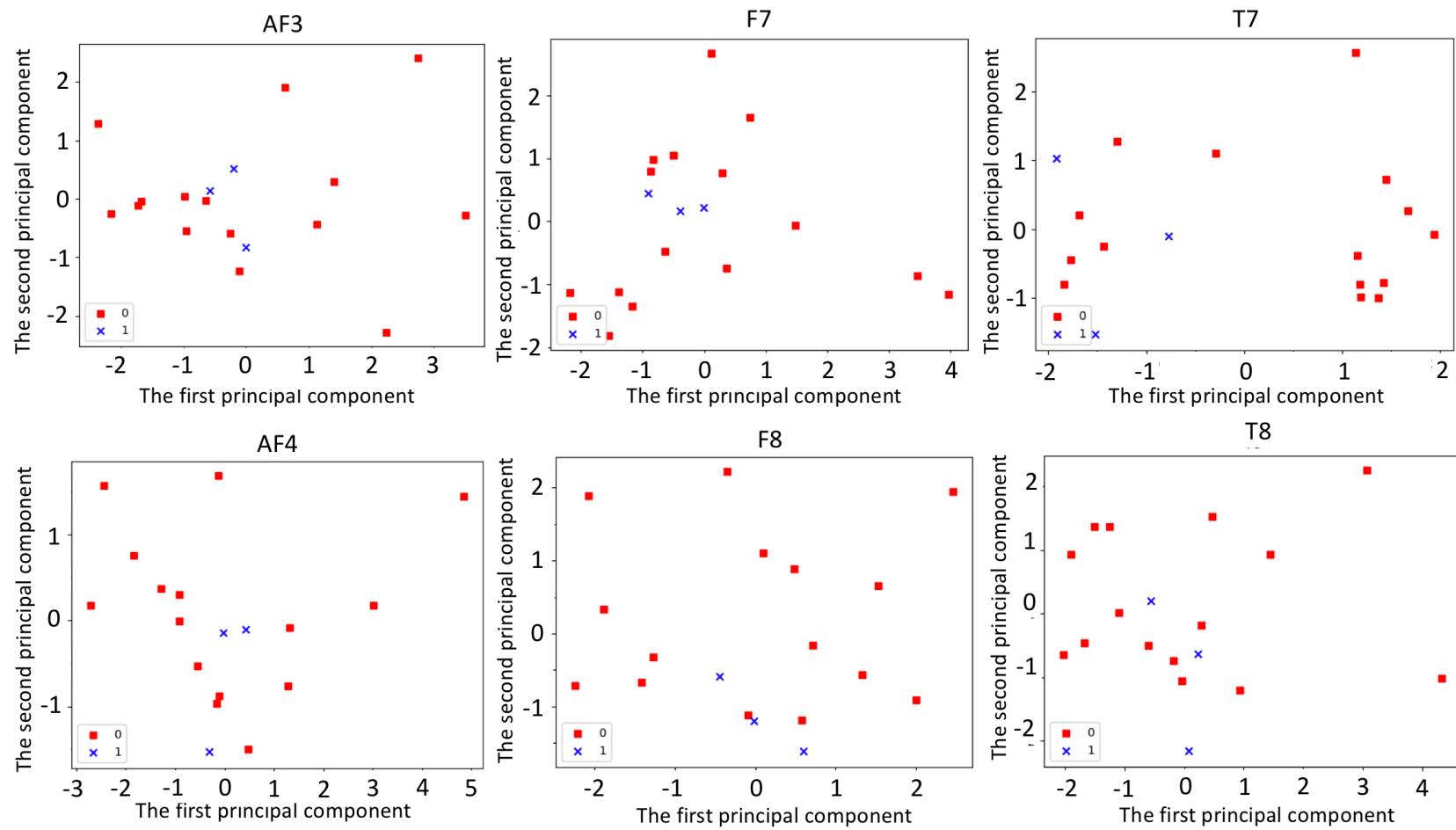
Table 3. Results of the pattern classifications.

\begin{tabular}{|c|c|c|c|c|c|c|}
\hline & AF3 & F7 & T7 & AF4 & F8 & T8 \\
\hline Subject A & $\frac{15}{20}=75 \%$ & $\frac{19}{20}=95 \%$ & $\frac{15}{20}=75 \%$ & $\frac{17}{20}=75 \%$ & $\frac{17}{20}=75 \%$ & $\frac{15}{20}=75 \%$ \\
\hline Subject B & $\frac{15}{18}=83 . \dot{3} \%$ & $\frac{17}{18}=95 \%$ & $\frac{15}{18}=83 . \dot{3} \%$ & $\frac{17}{18}=95 \%$ & $\frac{15}{18}=83 . \dot{3} \%$ & $\frac{15}{18}=83 . \dot{3} \%$ \\
\hline
\end{tabular}

Table 4. $\quad C$ and $\gamma$ at the highest accuracy.

\begin{tabular}{|c|c|c|c|c|c|c|}
\hline & AF3 & F7 & T7 & AF4 & F8 & T8 \\
\hline \multirow{2}{*}{ Subject A } & $C=0.1$ & $C=10$ & $C=0.1$ & $C=100$ & $C=0.1$ & $C=0.1$ \\
& $\gamma=0.1$ & $\gamma=58.0$ & $\gamma=0.1$ & $\gamma=2.5$ & $\gamma=0.1$ & $\gamma=0.1$ \\
\hline \multirow{2}{*}{ Subject B } & $C=0.1$ & $C=10$ & $C=0.1$ & $C=1000$ & $C=0.1$ & $C=0.1$ \\
& $\gamma=0.1$ & $\gamma=2.5$ & $\gamma=0.1$ & $\gamma=0.9$ & $\gamma=0.1$ & $\gamma=0.1$ \\
\hline
\end{tabular}

expounded again (shown in Fig. 9). It was judged as "Understanding" or "Non-understanding" by the following confirmation method. The confirmation is carried out on the basis of "answer is correct" and "can explain the process of problem". It is regard as "Understanding" if it satisfies all and "Non-understanding" otherwise. Table 2 shows the results of EEG measurements. "U" and "NU" represents "Understanding" and "Non-understanding" of the answers to the questions of whether understanding the exposition, respectively. The window width of the FFT was set to 128 points, and the hamming window was adopted as the window function. In the SVM, the parameters $C$ and $\gamma$ of the RBF kernel were grid-searched and the highest accuracy was calculated. $C$ was set to 5 patterns $(0.1,1,10,100,1000)$, and $\gamma$ was increased from 0.1 to 100 by 0.1 for each $C$. In order to evaluate the proposed method, the leave-one-out cross validation (LOOCV) method was used. Figs 10 and 11 are the results of the EEG features obtained using the PCA for the subjects A and B, respectively. Red and Blue symbols show a distribution of " $U$ " and "NU", respectively. The distributions of each channel are different in both the subjects $\mathrm{A}$ and $\mathrm{B}$. Then, we confirmed that the distributions of "NU" (blue) were small at F7. Table 3 shows the results of the EEG pattern classifications. We confirmed that the EEG pattern classification accuracy was the highest when the sensing position to record the EEG was F7 in both the subjects $\mathrm{A}$ and $\mathrm{B}$. Table 4 shows $C$ and $\gamma$ at the highest accuracy.

\section{Discussions}

In the experimental results, we obtained relatively good results. These results suggest that the EEG can check whether human understands contents of learning. In Table 3, the recognition accuracy of the EEG pattern classification was the highest when the sensing position was F7 in each subject. These results suggest that it is important to detect the EEG signals related to contents of learning because F7 is located in the lower frontal gyrus of the left brain and near the Broca's area. Moreover, the left brain can relate to numerical calculations. This means that different brain activities might be expressed with respect to numerical calculation from problems handled. The lower frontal gyrus is located at the lower part of the forehead. The forehead can relate to action decisions and memory matching. Therefore, the responses related to understanding of leaning might include the action decisions and the memory watching. The Broca's area relates to speaking words. The subjects answered the question with recording the EEG. Therefore, the activities of the EEG at F7 is unique.

\section{Conclusions}

In this paper, we proposed the method to check whether human understands contents of learning using EEG. The proposed method consisted of three stages; the EEG measurements, the EEG feature extraction and the EEG pattern classification. The EEG feature extraction method (stage) consisted of three phases; frequency analysis, band division and principal component extraction. The frequency analysis converted an original waveform of EEG into frequency components bands. In the band division, frequency components were divided into five rhythms $(\theta$, Low- $\alpha$, High- $\alpha$, Low- $\beta$ and High- $\beta$ waves). PCA calculated the first and the second principal components. The SVM classified "Understanding" and "Non-Understanding" on the basis of PCA results. In order to show the effectiveness of the proposed method, we conducted the experiments using real EEG data. In experimental results, we confirmed that the EEG pattern classification accuracy is the 
highest when the sensing position was F7. These results suggest that it is important to measure and use EEG signals if we judge whether understanding the contents of learning.

\section{References}

(1) Tobias Kaufmann, Andreas Herweg and Andrea Kübler: "To ward brain-computer interface based wheelchair control utilizing tactually-evoked event-related potentials", Journal of NeuroEngineering and Rehabilitation, DOI: 10.1186/1743-0003-11-7, January 16, 2014

(2) R. B. Braga, C. D. Lopes and T. Becker: "Round Cosine Transform Based Feature Extraction of Motor Imagery EEG Signals", World Congress on Medical Physics and Biomedical Engineering 2018, Vol. 2, pp.511-515, June 3-8, 2018

(3) Kenji Kato, Kensho Takahashi, Nobuaki Mizuguchi and Junichi Ushiba: "Online detection of amplitude modulation of motor-related EEG desynchronization using a lock-in amplifier: Comparison with a fast Fourier transform, a continuous wavelet transform, and an autoregressive algorithm", Journal of Neuroscience Methods, Vol. 293, pp.289-298, 1 January, 2018

(4) T. Inoue, K. Shinosaki, H. Sakamoto, S. Toi, S. Ukai, A. Iyama, Y. Katsuda and M.Hirano: "Quantification of EEG irregularity by use of the entropy of the power spectrum", Electroencephalography and Clinical Neurophysiology, Vol. 79, pp.204-210, September, 1991

(5) Nikolay N. Neshov, Agata H.Manolova, Ivo R.Draganov, Krasimir T.Tonschev and Ognian L. Boumbarov: "Classification of Mental Tasks from EEG Signals Using Spectral Analysis, PCA and SVM", Vol. 18, pp.81-92, 30 March, 2018

(6) Xiaoou Li, Xun Chen, Yuning Yan, Wenshi Wei and Z. Jane Wang: "Classification of EEG Signals Using a Multiple Kernel Learning Support Vector Machine", Sensors, Vol. 14, pp. 12784-12802, July, 2014

(7) Mohamad El Haj, Pascal Antoinev and Dimitrios Kapogiannis: "Flexibility decline contributes to similarity of past and future thinking in Alzheimer's disease", Hippocampus, Vol. 25, pp.1447-1455, November, 2015

(8) Florian Mormann, J uergen Fell, Nikolai Axmacher, Bernd W eber, Klaus Lehnertz, Christian E.Elger, Guillén Fernández: "Phase/amplitude reset and thetagamma interaction in the human medial temporal lobe during a continuous word recognition memory task", 